\title{
Control Network Reconstruction of Nickel Plates Packaging System
}

\author{
Zhao Zhengtian $^{1,2, a}$, Rui Zhiyuan ${ }^{1,3, b}$, Duan Xiaoyan, ${ }^{2,4, c, *}$ \\ ${ }^{1}$ School of Computer \& Communication, Lanzhou University of Technology, Lanzhou, China \\ ${ }^{2}$ School of Electrical \& Information Engineering, Lanzhou University of Technology, Lanzhou, China \\ ${ }^{3}$ Mechanical and Electrical Engineering College, Lanzhou University of Technology, Lanzhou, China \\ ${ }^{4}$ Key Laboratory of Gansu Advanced Control for Industrial Processes, Lanzhou University of Technology, \\ Lanzhou, China \\ azzt_1@163.com, bzhiy_rui@163.net, 'duanyan_0325@163.com \\ * Duan Xiaoyan
}

Keywords: Nickel Plates, Packaging System, Communication Failure, Profibus-DP, Subsystem, Control Network Reconstruction

\begin{abstract}
In this paper, an Automated Nickel Plates Packaging System, its functions, and its hardware architecture are introduced. This paper analyses the causes of the control system Profibus-DP communication failure in detail, and proposes a method of building subsystem, by partly giving up Profibus-DP, using TCP/IP communication among subsystems, and removing strong interference sources VFDs from the communication net. After control net reconstruction, the Automated Nickel Plates Packaging System has recovered its previous stability, and new control network brings equipment repair convenience.
\end{abstract}

\section{Introduction}

Nickel is a silvery white metal with good mechanical strength and ductility. Insoluble in water, strong resistance to acids and bases, but soluble in dilute nitric acid and nitro-hydrochloric acid. Resistance to high temperature, melting point $1455^{\circ} \mathrm{C}$, the boiling point of $2730^{\circ} \mathrm{C}$. The density was $8.902 \mathrm{~g} / \mathrm{cm}$ cubed. Nickel is often used in the production of stainless steel, alloy structural steel and other steel fields, electroplating, high-nickel base alloy, and battery fields, widely used in aircraft, radar, and other military manufacturing, civil machinery manufacturing, and electroplating industry.

At present, Famous Nickel enterprises include Russia MMC Norilsk Nickel, Brazil Vale SA, UK Xstrata Plc, UK Anglo American Plc, Australia BHP Billiton Ltd., and Australia Minara Resources Ltd. Jinchuan Group Co. Ltd. is the largest producer of Nickel in China and the second largest nickel producer in the world. Nickel plate is one kind of main nickel product form. Nickel plate packaging pipeline fashion at Jinchuan nickel smelter haves 8 stages. There are includes cutting ears, stacking, transferring, compressing, weighing, strapping, coding, and warehousing.

In this paper, we focus on the control system communication failure of nickel plates packing pipeline fashion at Jinchuan nickel smelter. After analyzed the fault cause, we reconstructed the control network. The actual production process is stable, the fault rate is low and the production efficiency has been raised. In Section 2, we present a nickel plate packaging pipeline fashion at Jinchuan nickel smelter. Section 3 gives the analyzing the failure in detail. Section 4 presents how the control network has been reconstructed. Section 5 summarizes the effectiveness of our proposal.

\section{Nickel Plate Packaging Pipeline}

We diagnosis communication fault on a manufacturing system called the Automated Nickel Plates Packaging System (ANPPS), shown in Figure 1. The primary purpose of this system is to take the original nickel plates, cut their ears, stack them in one block, compress them, weight them, 
strap them, print code on them, and put them in storage. The whole process can be divided into 3 stages, including cutting original plates' ears, transfer blocks, and package.

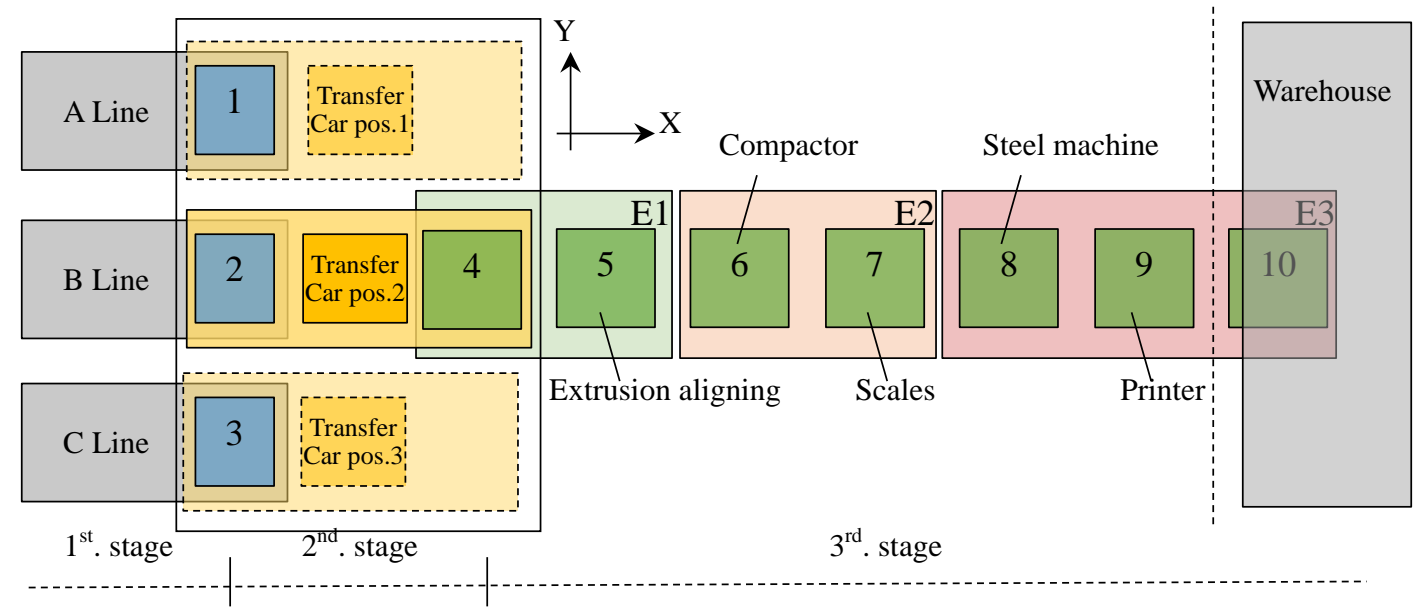

Figure 1 An overall system diagram for the Automated Nickel Plate Packaging System (ANPPS)

\subsection{Cut Original Plates’ ears}

Original nickel plate which length: 900-920mm, and width: 880-900mm, has 2 ribbon hung handle which like 2 ears, shown in Figure 2.ANPPS has 3 line (A Line, B Line, and C Line, shown in Figure $1,1^{\text {st }}$.stage) to cut original nickel plates' ears meanwhile, and stack nickel plates without ears, shown in Figure.3. A block of nickel plates high: 300-700mm.

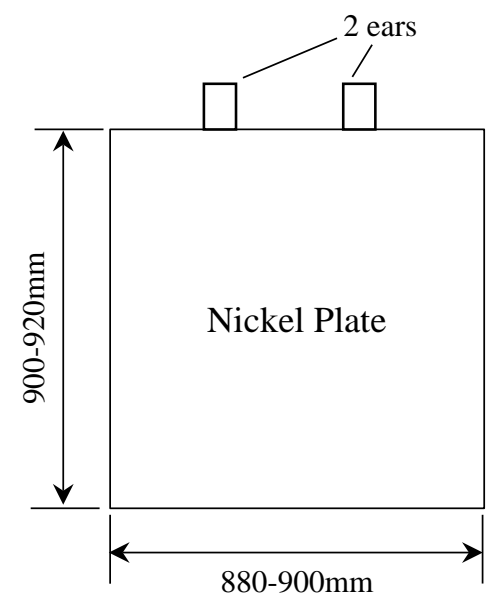

Figure 2 Original Nickel Plate

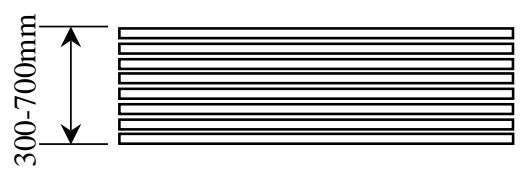

Figure 3 A block of Nickel Plates

\subsection{Transfer Blocks}

When 3 Line finished stacking, transfer car move the blocks of nickel plates from position 1, 2, and 3 to position 4 . It is $2^{\text {nd }}$. Stage. The transfer car is driven by 2 VFD motors. First the transfer car could move along Y-axis to A, B, and C lines' position, second move in the negative direction of the $\mathrm{X}$-axis to 1,2 , and 3 positions loading the blocks, then take the blocks to position 4 . When the blocks reach position 4 , it would enter $3^{\text {rd }}$. stage, packaging.

\subsection{Package}

The $3^{\text {rd }}$. stage has 3 chain conveyors E1, E2, and E3, which are driven by 3 VFD motors. The blocks move along the positive direction of the X-axis by the chains. The blocks is extruded along the $\mathrm{X}$-axis and Y-axis at position 5 by a pear of hydraulic machines. It make each piece of block aligned, like a cube. Because of Plate bending, the blocks are compressed at position 6 by compactor which is driven by hydraulic. It make each piece of block closely proximity to each other. The blocks are weighted for deal at position 7. There are 2 scales stacked up and down. The blocks are packaged by a steel machine at position 8 , and printed the code at position 9 . The code displays 
product name, standard, bantch No, grade, date, net weight, gross weight, and package No. When the blocks reach position 10, it would be put in storage According to the code by warehouse.

\section{Communication Fault Analysis}

\subsection{Communication Fault Phenomenon}

ANPPS was completed and put into production in 2014. It was initially running smoothly, however, System prompts warning of communication fault irregularly and continually since 2015. When the communication fault occurs in control system, it always follows key data loss, frequently shutdown and restart of oil pump motor, and compactor recovery difficulty. The failure rate of communication is up to 2 times per minute. The ANPPS can not be stable, continuous operation. The nickel plates production is delayed seriously. Unstable nickel output of Jinchuan Group Co. Ltd. even affected the price of nickel on the Shanghai futures exchange.

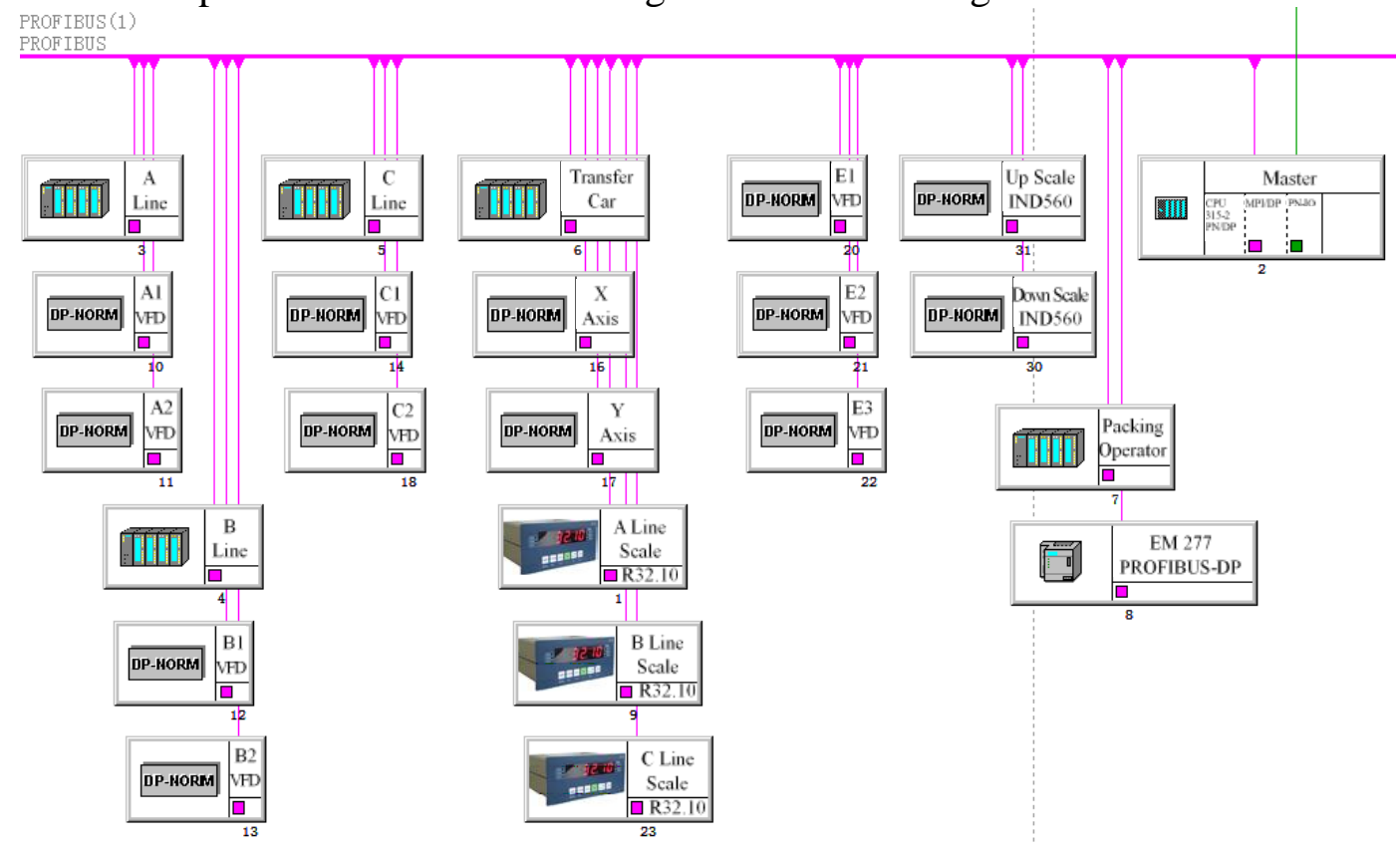

Figure 4 Profibus-DP Topology

\subsection{Hardware Architecture}

Control system of ANPPS is based on Programmable Logic Controller(PLC). Because of 1236 digital inputs, 557 digital outputs, and 6 analog inputs, ANPPS uses medium sized PLC. The PLC's CPU model is 315-2 PN/DP which belongs to SAMTIC S7-300 series. There are 5 remote IO ET200 and 5 touch panels for A Line, B Line, C Line, transfer car, and packaging. There are 11 VFDs, and their model is ABB ACS800, which drive motors for E1, E2, E3, X-axis and Y-axis of Transfer Car, 3 pairs chains of load and remove which belong to A Line, B Line, and C Line. There are 5 scales. Three of them is for A Line, B Line,and C Line, which is used to measure the weight of the blocks.Two of them is used for deal. The Printer'controller is CPU226 with EM277 communication module. All of these devices support profibus-DP communication. The Profibus-DP Topology is shown in Figure 4. The real connection path of Profibus-DP is shown in Figure 5. 


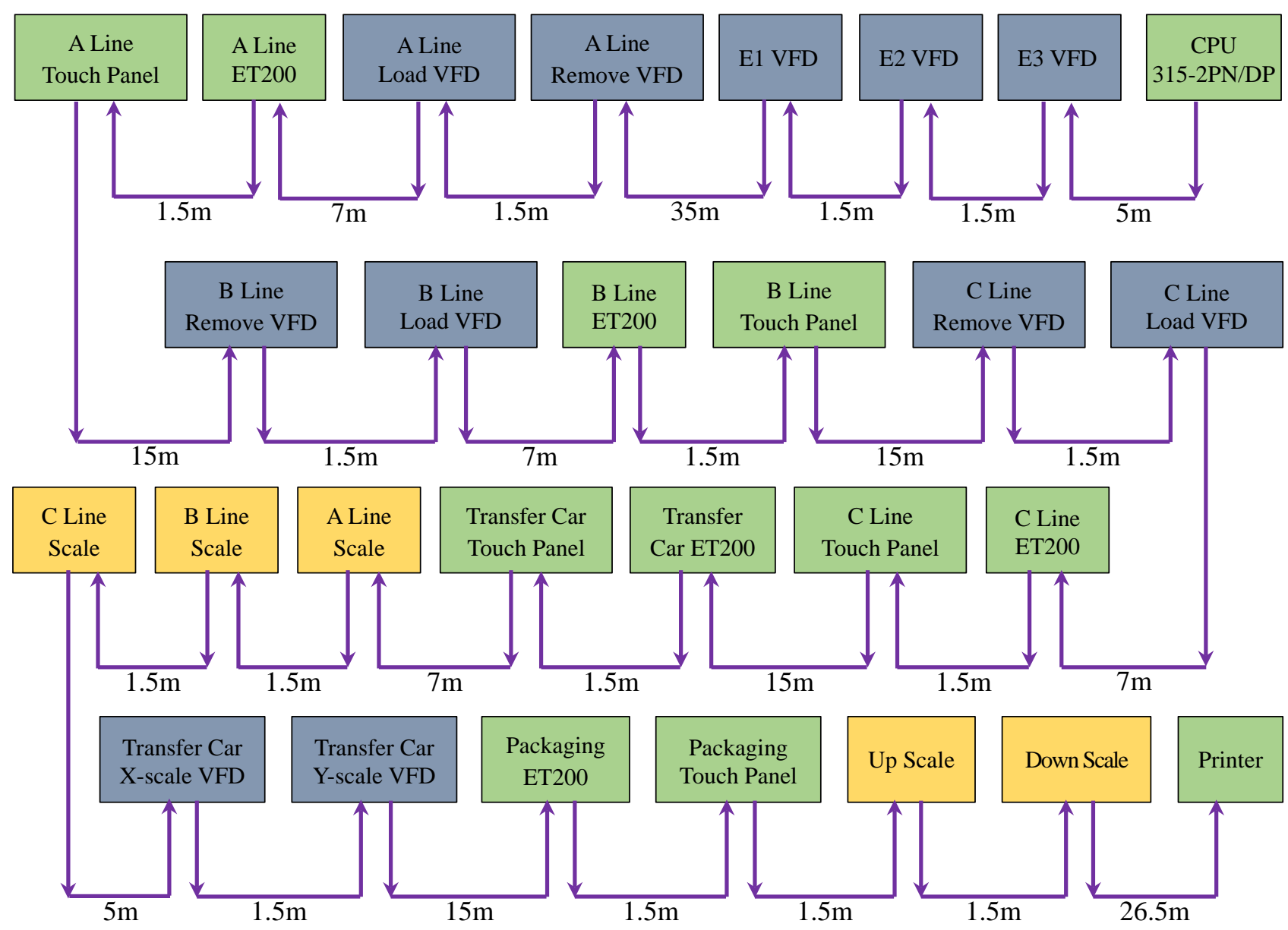

Figure 5 Real Connection Path of Profibus-DP

\subsection{Failure Cause Analysis}

The real connection path of Profibus-DP is typical series topology. Because the transfer rate of 5 scales is limited, transfer rate of ANPPS is only $1.5 \mathrm{Mbps}$. However, the length of path is $182 \mathrm{~m}$, which is close to $200 \mathrm{~m}$ distance limitation when transfer rate is $1.5 \mathrm{Mbps}$. Additionally, due to the negligence of the control system designer, communication line of Profibus-DP to power equipment and to weak current equipment was not laid separately. Through measurement, the Profibus is grounded undesirable. All of these reasons could lead to communication interference.

\section{Control Network Reconstruction}

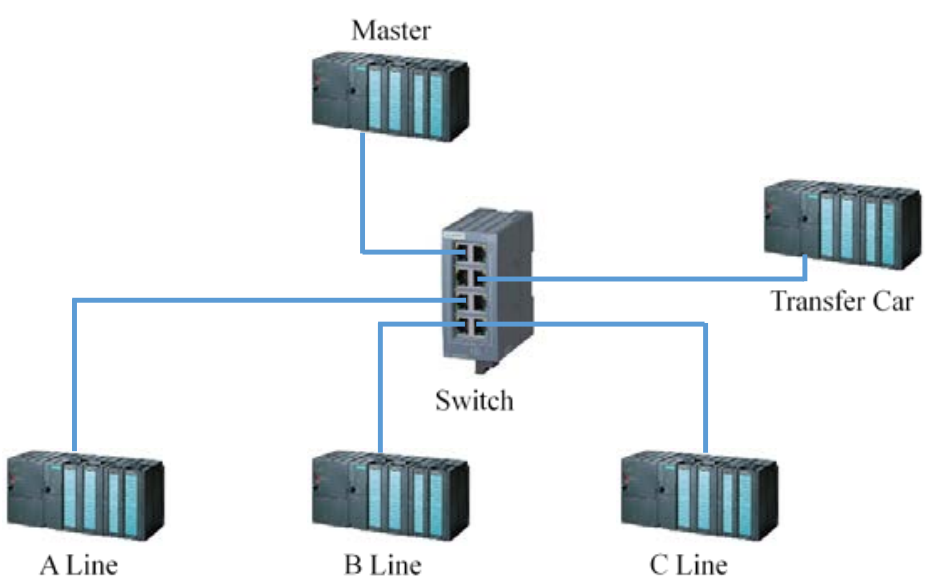

Figure 6 New System Topology

We proposed an approach of building subsystem to solve the failure. Firtly we finded that the 
functions of cutting ears and stacking can be independent of other functions, when they are finished, they just have to wait the Transfer Car. Secondly the function of the Transfer Car is also independent of other functions, it only move the blocks from position 1 or 2 or 3 to the position 4, it is always waiting A Line or B Line or C Line finished in most of the time. Then once the blocks reach position 4 , the operations like compressing, weighing, strapping, coding, and warehousing must be completed in turn. So A Line, B Line, C Line and Transfer Car could be split into 4 subsystem. The other part of packaging line is an integral whole.

\subsection{Building subsystem}

We replace ET200 of A Line, B Line, C Line, and Transfer Car with CPU315-2PN/DP. The new system topology diagram is shown in Figure 6. Obviously, it's star topology. We retain the original IO address and programs, partly give up the Profibus-DP communication mode, and use TCP/IP communication among subsystems by a 8 port switch which model is SAMTIC SCALANCE XB008. All VFDs use IO control mode by adding IO module, and removed from Profibus-DP. Inside the Transfer Car subsystem, Profibus-DP communication mode is used to exchange data between CPU and 3 scales. The other 2 scales, ET200 of Packaging, Printer CPU(connected by EM277), and Master CPU are also connected by Profibus-DP. The reconstructed control system is shown in Figure 7.

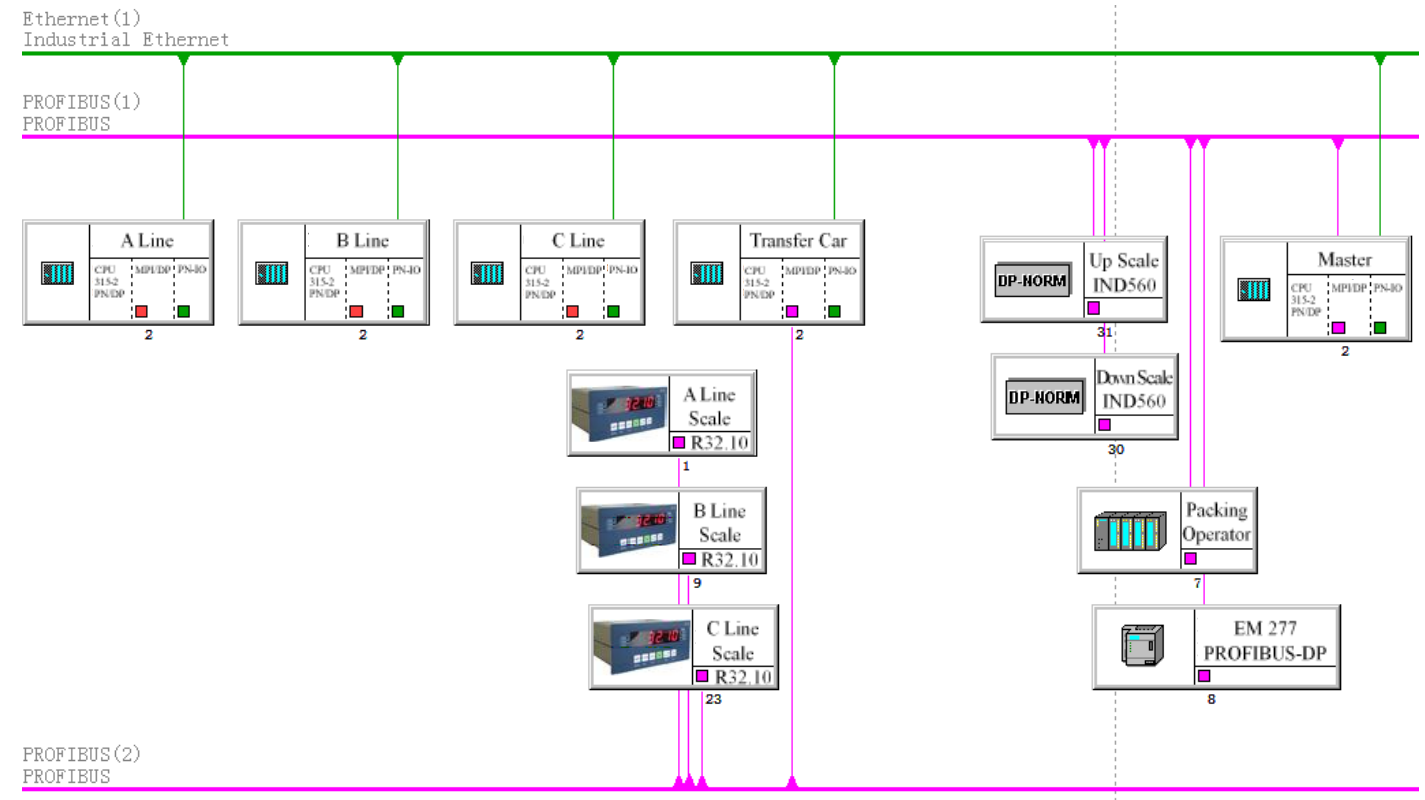

Figure 7 Reconstructed Control System

\subsection{Key Issue}

When control system has been reconstruted, the key issue which ensures the program runs properly is how to exchange data among subsystems by TCP/IP communication. We have achieved by following 3 steps. Firstly we storage the exchange data to some DB blocks according to different exchange cycles. Secondly we have programmed pulses of different frequencies shown in Figure 8. We could adjust the TV (Time Value) of 2 S_ODT blocks to change the pulse dutyfactor. Then we use FB15 to send data among subsystems shown in Figure 9. The M1.6 on the REQ of FB15 is just our programmed Custom Pulse.The ID is a serial number of S7 connection between master and other subsystems. 


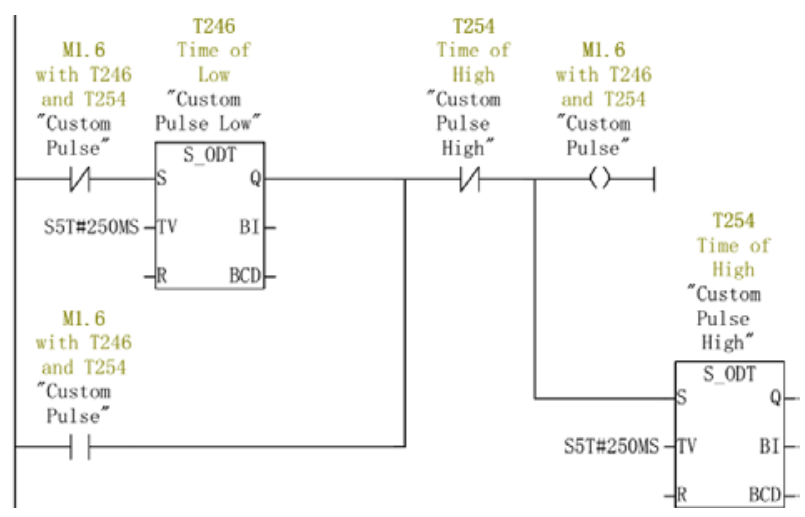

Figure 8 Pulse Ladder

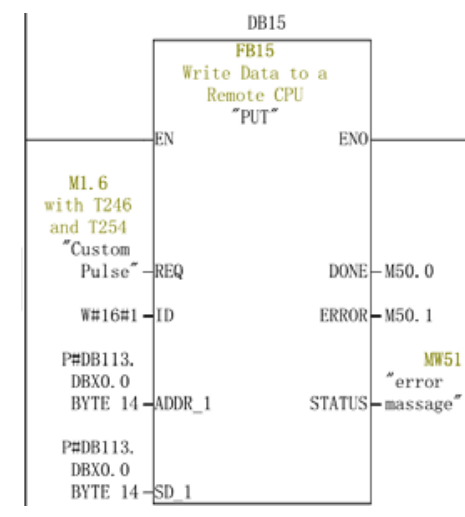

Figure 9 Send Data Ladder

\section{Conclusion}

In this paper, we presented Automated Nickel Plates Packaging System (ANPPS), its functions, and its hardware architecture. We analysed the causes of the original system Profibus-DP communication failure in detail, proposed a method of building subsystem, by partly giving up Profibus-DP, using TCP/IP communication among subsystems, and removing strong interference sources VFDs from the communication net. After control net reconstruction, the ANPPS has recovered its previous stability. According to statistical analys,the rate of communication failure dropped from the highest 2 times per minute to 2 times per week. Especially, when any one of A Line, B Line, and C Line stopped to repair, other parts of ANPPS could continually operate without any impacts.

\section{Acknowledgements}

In this paper, the research was sponsored by the National Natural Science Foundation of China (Grant No. 51865027), Emerging Engineering Project of the Ministry of Education of PRC (Construction and Exploration of Robot Specialty in the Context of Emerging Engineering), and Youth Science and Technology Foundation of Gansu Province (1506RJYA103).

\section{References}

[1] http://www.insg.org/recycling.aspx

[2] Fieldbus Technology Handbook on PROFIBUS-DP. SIEMENS Corporation. 1999.

[3] DP/DP Coupler User Description Version V1.5. SIEMENS Corporation. 2000.

[4] https://support.industry.siemens.com/cs/document/8859629.

[5] https://support.industry.siemens.com/cs/cn/zh/view/62543517.

[6] The ACS800 firmware manual. ABB Corporation. 2010. 